\title{
A Clinical Research Tool that can Decrease Pharmaceutical Study Costs, Improve Subject Recruitment and Increase Patient Retention and Adherence
}

\author{
Article by Nicole C. Hank, \\ Perseverance Research Center, LLC \\ E-mail:nhank@prcresearcheducation.com
}

\begin{abstract}
Clinical trials are a key research tool for advancing medical knowledge and patient care. They ultimately gear towards ameliorating and improving diagnosis and treatment of all diseases by introducing novel interventions and therapies. Essential to conducting a clinical trial is randomizing the proper study population through recruiting eligible participants based on inclusion and exclusion criteria, and ensuring study completion and adherence of study patient participation. Although these may seem simplistic, patient recruitment, retention and adherence are the most difficult challenges in clinical trials. While patient recruitment is one of the key elements to study completion, over $80 \%$ of clinical trials shockingly do not finish on time. Devising a potential strategy is vital in overcoming recruitment barriers; however, it is very costly and cumbersome. Lambda Health, LLC is in the process of creating a clinical research tool that can interface with EMR, in hopes of increasing patient retention through proper site selection, and setting reminders to patients about medication dosing, diary documentation, study procedures and visits scheduling. Ideally through implementing this tool into clinical trials, less effort and money will be spent on recruitment and the rates of adherence and retention will increase.
\end{abstract}

Keywords: Patient recruitment, retention rates, patient adherence, cost-effective, clinical research tool.

\section{Introduction}

The key determinants of a successful clinical trial are based on the success or failure of recruitment and retention of study participants. Most clinical trials fail to meet recruitment goals, which in turn, leads to study delays, early trial termination, increased study costs, or inability to draw conclusions at trial completion due to loss of statistical power. A study recently conducted by Carlisle, et. al (2015) found that $19 \%$ of registered trials that closed or were terminated in 2011, either failed to meet accrual goals and an alarming $86 \%$ of all U.S. clinical studies fail to recruit the required number of subjects on time. When a study struggles with study patient recruitment, the scientific and financial viability is significantly impacted. It can create uncertainty about the efficacy of a treatment, which can then delay bringing a potentially effective therapy to market. Slow acquisition of study patients may also impact financial investments of agencies funding a study, possibly leading agencies to suspend or even terminate the study and fund less reliable but more rapid recruiting studies. Since poor recruitment is a crucial factor in conduct in all clinical trials, pharmaceutical companies have spent billions of dollars investigating the barriers to recruitment and devising potential strategies such as piloting the recruitment process, financial and educational incentives for clinicians, newsletter and reminders for patients, amending study protocols, assisting with patient travel, and networking with various healthcare professionals. In the United States, the average cost per patient, between the years 2008-2013, had increased by $157 \%$, according to Medrio data (http://medrio.com/partners/the-top-5-cost-drivers-in-phase-i-clinical-trials/2015). These costs have been attributed to subject recruitment to avoid early study closure or trial failure. Participant recruitment to clinical trials has been called "the most difficult and challenging aspect of clinical trials," (McDonald, 2011) with flaws in recruitment identified as one of the main reasons for the failure of clinical studies. 
DOI: $10.21522 / \mathrm{TIJCR} .2014 .04 .02 . A r t 003$

ISSN: $2520-3096$

\section{Study recruitment}

Recruiting patients into a clinical trial takes careful planning and flawless execution from multiple key players. Although the site is often responsible for contributing the majority of patients needed to meet enrollment targets, several study personnel are ultimately responsible for patient recruitment, which essentially starts with site selection. Although Sponsors feel confident in their selections based from site feasibility questionnaires and site initiation visits; a site or PI may not be able to recruit patients as needed and required by a Sponsor. Poorly performing sites has long been a challenge for the industry and increasing protocol complexity has created new challenges with feasibility. According to research from FUFTS CSDD nearly 40 percent of all amendments occur before the first study volunteer receives first dose (http://csdd.tufts.edu/news/complete_story/pr_ir_jan_feb_2016) mostly because of slow enrollment. In fact, it has been determined that most of investigative sites under enroll, $11 \%$ of sites fail to enroll a single patient and only13\% exceed their enrollment target (FUFTS CSDD, 2013 http://csdd.tufts.edu/news/complete_story/pr_ir_jan-feb_2013). These recruitment and retention problems create not only major delays and increased pharmaceutical costs, but also delay approval in a potential effective, ground breaking therapy. With augmentation of study costs and extensive time and effort spent in the process of clinical trial recruitment, improving the rate of recruitment and retention is warranted. The industry has begun to recognize that there is an issue, pouring an increasing amount of money into the process but seeing results plateau. TUFTS Center for the Study of Drug Development (2014, http://csdd.tufts.edu/news/complete_story/pr_tufts_csdd_2014_cost_study) concluded that total capitalized cost per approved new compounded grew at an $8.5 \%$ compound annual rate while rates of drug approval, in relation to cost, dropped significantly. If increased funding is not the answer to recruitment then what is? Although there isn't one specific answer for study costs increasing, delays in study trial completion, and therapies not being approved, new strategies have been implemented by pharmaceutical companies.

Social networking like Facebook and Twitter not only help companies spread the word about a specific trial but assist with reducing the cost per patient while targeting specific demographic populations (Clinical Trial Media, 2011). Social media has been an effective method for generating pre-qualified patient referrals and lowering the overall cost per randomized patient. In 2009, Acurian, a leading provider of clinical trial patient recruitment and retention solutions for the life sciences industry, generated $54 \%$ of their prequalified patient referrals through social networking channels and proprietary online health channels -(June 2011 http://www.healthcaretrendsnewsletter.com/2010/07/social-media-for-patient-_recruitment-only-thebeginning/).

In addition to social networking, sponsors have also been working with online communities such as Patients Like Me and Clinical Trial Awareness, which allow patients to monitor and submit data on the effects of novel drugs. This has opened new ways of testing treatments and accelerating patient recruitment into clinical trials as well as reducing the cost of conducting a clinical trial (Brownstein et al, 2009). In addition to social media, and online community marketing, pharmaceutical companies have also created Clinical Research Recruitment Specialist positions for primarily assisting sites with patient recruitment. These specialists typically visit sites, assist with additional marketing materials and provide sites with additional funding for radio, TV and other marketing avenues. While this has been productive and successful, this can be quite costly. So why is recruiting patients for clinical trials such an arduous, expensive task? Some sites believe study designs are getting too difficult to recruit patients, while others believe sites are inaccurately estimating how many eligible patients they have and how many they can enroll. Regardless of the reason, a new, improved, less costly strategy needs to be implemented.

\section{Feasibility}

In clinical research, feasibility is the precursor to site selection. Before a site is even selected to participate, sponsors provide feasibility questionnaires that are completed by a potential site. Recruitment factors including proper patient population of the disease of interest, determining subject eligibility, listing of competitive enrolling trials, estimating total number of subjects a site can enroll and in what time frame, 
are typically the standard questions asked in a feasibility questionnaire. Assessing these and other factors allow a sponsor to choose which sites are most appropriate for a particular clinical research study, and who may be the most successful in recruiting based on site responses. Estimating enrollment potential for a clinical trial is a typical part of the feasibility process; however, patient enrollment remains one of the greatest challenges in the clinical research industry. In fact, most trials end up doubling their original timelines to meet enrollment goals, and $48 \%$ of sites under-enroll study patients (Tufts, 2013). Sponsors go through a thorough feasibility process to ensure a site is adequate and has the large patient population to enroll patients from, yet there are still issues with patient recruitment. If a site inaccurately or overestimates subject eligibility, this could lead to unsuccessful trials. Accurately reporting a sites patient population during the feasibility plays such a large role in site selection since approximately $80 \%$ of study patients are utilized from within a site's own database and the remaining $20 \%$ are typically recruited from various outreach methods. (Centerwatch, 2016). As expected, a site's principal investigator and the study coordinator are crucial to patient recruitment. After a site is selected, one of the first steps of recruitment is searching within the site's database. However, if a site overestimates their ability to recruit study patients, they will spend extensive time and effort prescreening. Once a site finds a patient who may be eligible, the potential patient must then be contacted and educated about the study and properly consented. Once a patient is identified, consented and enrolled in the study, it is also the site's responsibility to ensure the patient is always updated and made aware of appointments, study procedures, and drug dosing to ensure patient adherence and retention.

\section{Patient retention}

Adequate subject enrollment also provides a foundation for projected subject retention which is a vital component in evaluating patient data. Although, recruitment is a major issue and one of the largest issues plaguing clinical trials, retention and adherence are just as critical. In reality, retaining study patients from start to finish can be just as challenging as patient recruitment. Clinical trials face serious consequences from dropouts through study delays, increased study costs, as well as missing data that essentially compromises the results and integrity of a study. According to a recent study, dropout rates are between 30-40\% in clinical trials (Alexander, W. 2013). Although it is a patient's right to drop out from a trial, for any reason, at any given time, which is thoroughly explained in the informed consent process, there are many reasons why patients withdraw. Despite good intentions of finishing a study, many participants end up dropping out for a variety of reasons. While some dropouts are due to uncontrollable circumstances, others are preventable. Of course, subjects have the right to discontinue participation in research at any time. However, more can be done to prevent withdraws and lower dropout rates.

\section{Communication issues}

Even with pharmaceutical companies spending billions of dollars in recruitment, withdrawal of patients from clinical studies is not an uncommon phenomenon and is inevitable in some circumstances. Lack of efficacy of the drug being tested, the occurrence of adverse and intolerable side effects, lack of patient follow-up, or simply patient withdrawal of consent during the clinical trial are some reasons encountered (Gabriel \& Macodo, 2011). Although patient retention is the strategy and tactics designed to keep patients enrolled in clinical trials, and from discontinuing participation and dropping out, patient adherence, which is a level of compliance a patient has with taking their medication as prescribed, is just as important. Today, the average trial adherence rates are only 43-78\% (Zonana, 2015) which is a main reason why most clinical trials fail. Some patients may simply forget while some may not have a clear understanding of the importance of taking medication properly or finishing a trial. Data from noncompliant patients can affect trial results to such an extent that they can make or break a drug making it to market, thus why it is imperative that sites need to create a strategy for better medication adherence. 
DOI: $10.21522 / \mathrm{TIJCR} .2014 .04 .02 . A r t 003$

ISSN: $2520-3096$

\section{How to improve recruitment, retention and adherence}

Improving both patient recruitment and retention can be achieved through better site and patient communication. The aim of fostering strong links between pharmaceutical companies, clinical trials and potential patients is obviously a concerted goal for all involved in the process. The question, of course, is how can this aim be achieved and how can the number of patients recruited and retained be increased? Through creating a Tool that can be utilized among all sites and implemented into EMRs, and into smart phones, can open new lines of communication as well as strengthen those that already exist. Let's start with initial feasibility of a site. If a site was able to accurately and properly estimate how many patients at their site may be eligible to participate, this can help sponsors accurately chose a site during site selection, leading to increased patient recruitment, and lowered costs spent on unnecessary recruitment strategies. Although patient retention can be out of our control due to uncontrollable circumstances, if patients had better communication with site personnel, this may help with patients wanting to remain in a study. Since patients tend to forget when they should take their medications, when to fill out their diary, or even remembering when their study visit is, if they had constant reminders on their phone, this could improve patient retention and adherence. Lambda Health, LLC, is in the process of creating a clinical research tool that could integrate inclusion and exclusion criteria into multiple EMR systems through importing HL7 data into a database and apply faceted search and analytics across the data based on specific feasibility questions.

\section{Methods}

Lambda Health, LLC is in the process of developing a tool that would be used strictly for clinical research to accommodate studies of different interventions or disease states. This tool will have the capability of matching eligibility criteria to relevant data fields and flagging potential trial subjects to investigators. It will also be able to provide a pharmaceutical company with more accurate estimate of patients that may be eligible to participate. Ideally patient questionnaires and surveys can be linked to can also be linked in the EMR to provide additional context to clinical data. This tool with also be utilized as a portal, which will only be utilized and accessed by each site's clinical team. This portal will provide updates and communication between the clinical staff and the patient. In addition, this clinical research tool will have the capability to be utilized as a phone application, utilized by study patients to keep track of study timelines, procedures, and any pertinent study information. Once a patient in consented and is randomized (enrolled) into a study, a member of the site's clinical team will register the patient into the study and provide them a subject number and access code. The patient, with the help of the site's clinical team, will be able to download the app from the app store and sign in with the provided access code. Only the designated members of the site's clinical team and the patient will have access to this information, and the patient will only be identified and registered with a subject number. Once a patient has successfully been registered into the app, they will be able to receive updates, access pertinent study information that pertains to them, and contact the clinical team with any inquiries. This clinical application will serve as a guide to the patient throughout the course of the study, reminding the patient when to take their medication, clinic visit dates and times, change in dosing schedules, prohibited medications, as well as an online tool to track adverse events and log concomitant medication in real-time. Through this constant patient interaction, eliminating missed visits, improper medication dosing, and keeping track of real time adverse events and concomitant medications, it is hopeful that through this clinical research tool, data collection will be streamlined and patient retention and study adherence will exceed pharmaceutical companies' expectations. This clinical research tool is currently being conducted in a local Investigator Initiated Trial in Scottsdale Arizona. Data and effectiveness and find tuning of this tool is anticipated to be released in late 2018.

\section{Regulations}

Lambda Health has experience with building mobile apps and mobile-specific UI/UX design that can achieve HIPAA and other compliance standards in technical implementation as well as has adequate healthcare domain expertise and experience with knowledge of HIPAA compliance. With this tool, Privacy 
issues and information governance are among the most complex aspects of implementing such a tool for clinical research. All data will be encrypted and secure to follow HIPAA guidelines. Patients will have a unique subject ID and unique password that only they will know. The only personnel that has access to the portal are members of the site's study team that will also have their own username and access code.

Since this is a new concept, we anticipate all study sites would need to consent their patient population regarding how their EMRs will be used and by whom. Language regarding a phone app would be integrated into the informed consent process that will have to be IRB approved. Developing optimal procedures for ensuring patients that are informed and protected, balanced with minimizing barriers to research is a major consideration as technology in clinical research advances.

\section{Discussion}

In clinical research, patient retention is a key factor in ensuring the success of a study; however, it is often overlooked and undervalued. Most of the time, effort and pharmaceutical costs are spent on patient recruitment and not retention. Poor clinical trial recruitment and retention is therefore likely to impede the successful evaluation of new and existing interventions, and prevent greater efficiency in clinical development. Keeping patients in clinical trials to ensure successful and long-term data-gathering requires careful planning and pro-active strategies. Attention needs to be directed to the individual needs of patients and site staff in relation to specific demands of the research protocol. Some patients may simply forget while some may not have a clear understanding of the importance of taking medication properly or finishing a trial. These are issues that can be aided by proactively planning strategies to educate patients on the importance of complying with all components of clinical protocol, to include medication instructions along with the value of completing a clinical trial. If sites had the capability to accurately report their study population and study eligibility, pharmaceutical companies wouldn't need to spend an obscure amount of money on recruiting strategies. Through implementing a clinical tool that can be implemented into a myriad of EMRs for site feasibility as well as has the capability of sending text message reminders to study patients, is an easy, inexpensive way to help patients remember to take medication and to remind them of upcoming study visits, therefore increasing retention and adherence.

\section{Conclusion}

Clinical research tools are a promising resource to improve the efficiency of clinical trials and to capitalize on novel research approaches. Through utilizing EMRs as useful data sources to support comparative effectiveness research and new trial designs that may answer relevant clinical questions as well as improve efficiency and reduce the cost of recruiting in clinical trials. Initial experience and early testing on such a clinical research tool has been encouraging, and accruing knowledge will continue to transform the application for clinical research. The pace of technology has produced unprecedented analytic capabilities, but these must be pursued with appropriate measures in place to manage security, privacy, and ensure adequacy of informed consent. Whether such a clinical research tool can be successfully applied to the conventional drug development in clinical trials remains to be seen and will depend on demonstration of data quality and validity, as well as realization of expected efficiencies. While there are no easy answers when dealing with both study retention and patient adherence, a clinical application that can interface with a sites electronic medical records strategies and tactics that address the major hurdles patients face to comply with the protocol, as well as educate patients on the expectations and assure their understanding of a clinical trial is essential. Through constant communication and engagement, the importance of taking study medication as directed and attended regularly scheduled study visits will hopefully create higher patients retention rate as well as higher adherence rates. 
DOI: $10.21522 / \mathrm{TIJCR} .2014 .04 .02 . A r t 003$

ISSN: 2520-3096

\section{References}

[1].Alexander, W. (2013). The Uphill Path to Successful Clinical Trials: Keeping Patients Enrolled. Pharmacy and Therapeutics, 38(4), 225-227.

[2].Carlisle, B., Kimmelman, J., Ramsay, T., \& MacKinnon, N. (2015). Unsuccessful Trial Accrual and Human Subjects Protections: An Empirical Analysis of Recently Closed Trials. Clinical Trials (London, England), 12(1), 7783.

[3].C. Brownstein, J.S. Brownstein, D.S. Williams, P. Wicks, and J. Heywood, "The Power of Social Networking in Medicine," Nature Biotechnology 27, 888-890, 2009.

[4].Centerwatch (2016) http://www.centerwatch.com/news-online/2016/06/27/issue-patient-retention-clinical-trials/ June 2016.

[5].Clinical Trial Media (2011), available at: http://www.centrewatch.com/professional/prv295.html.

[6].Gabriel, A. P., \& Mercado, C. P. (2011). Data retention after a patient withdraws consent in clinical trials. Open Access Journal of Clinical Trials, 3, 15-19.

[7].G. MacDonald, Social Media Boosting Patient Recruitment, but FDA Guidance Needed, March 2011, available at: http://www.outsourcing-pharma.com/Clinical-Development/Social-media-boosting-patient-recruitment-but-FDAguidance-needed Accessed 24 Jun 2011.

[8].Medrio data (http://medrio.com/partners/the-top-5-cost-drivers-in-phase-i-clinical-trials/2015).

[9].Social Media for Patient Recruitment Only the Beginnin (2011) http://www.healthcaretrendsnewsletter.com/2010/07/social-media-for-patient- recruitment-only-the-beginning/.

[10]. Tufts Center for Drug Development, 2016. Protocol Amendments Improve Elements of Clinical Trial Feasibility, But at High Economic and Cycle Time Cost http://csdd.tufts.edu/news/complete_story/pr_ir_jan_feb_2016.

[11]. Tufts Center for Drug Development, (2013) New Research from Tufts [CSDD] Characterizes Effectiveness and Variability of Patient Recruitment and Retention Practices.

[12]. Zonana M. (2015) How Poor Medication Adherence Is Damaging Your Clinical Trial Data and How to Fix it, Fast. 